\title{
Extra echo spaces: ultrasonography and computerised tomography correlations
}

\author{
TAKASHI WADA, MORIHIRO HONDA, SEIYA MATSUYAMA \\ From the Department of Medicine, International Goodwill Hospital, Yokohama, and the Department of Radiology, \\ Tokai University, Kanagawa, fapan
}

SUMMARY Among the echocardiograms of 844 patients of the International Goodwill Hospital from January 1980 to April 1981, 700 showed clinically inexplicable extra echo spaces. Fifty of the 700 had computerised tomography of their hearts which showed the extra echo spaces to be caused either by anterior or posterior subepicardial fat. Six of the $\mathbf{5 0}$ cases had both fat and pericardial effusions, which are difficult to differentiate echocardiographically unless follow-up clinical observations are performed. Subepicardial fat deposits are reasonable explanations for the extra echo spaces frequently observed by echocardiography: they correlate well with clinical findings. Subepicardial fat should be recognised as the cause of such extra echo spaces.

Echocardiography has contributed to the clinical diagnosis of pericardial effusions ever since the latter were quantitatively evaluated according to the sizes and characteristics of their "echo-free spaces". 1 It was also postulated that, in some cases, small amounts of pericardial fluid could be detected by echocardiography, even fluid in physiological quantities in normal subjects. Is echocardiography sufficiently sensitive to detect such small amounts of pericardial fluid? Can such small quantities of fluid actually produce images showing several millimetres separation of the epicardium from the pericardium posteriorly, permitting echocardiographic assessments?

The hypothesis that "echo-free spaces" represent pericardial effusions is based on the assumption that a dense, broad, band-like echo behind the posterior wall of the left ventricle is the pericardial echo, and that the curvilinear echo above the "echo-free spaces" is that of the epicardium. The epicardium consists of a single layer of mesothelial cells ${ }^{2}$; the pericardium is about $1 \mathrm{~mm}$ thick. ${ }^{3}$ Therefore, it is reasonable to assume that the epicardium alone cannot produce a curvilinear echo and that the pericardium alone is not likely to produce a dense, broad, band-like echo on echocardiography. Furthermore, "echo-free spaces" have not been clearly defined. In asymptomatic persons, inexplicable extra echo spaces are often identified behind the posterior wall of the left ventricle, which may be relatively echo free or even echo

Accepted for publication 10 December 1981 dense on comparison with the density of the myocardium. Using an automatic gain control or proper gain damping, however, these spaces usually become echo free. Were we to assume that all such extra echo spaces behind the posterior wall of the left ventricle are the result of pericardial effusions, the frequency of pericardial effusions would appear to be much greater than we know it to be. Furthermore, our experience indicates that in many cases, these extra echo spaces do not correlate with the clinical findings of pericardial effusions, and they fail to respond to treatment during lengthy follow-up studies (Fig. 1).

The question arises as to what these extra echo spaces represent and why they are so frequently observed echocardiographically. In some instances preoperatively encountered posterior extra echo spaces cannot be confirmed at operation as being caused by pericardial effusions. Identical extra echo spaces continue to present themselves in uncomplicated postoperative patients. Extra echo spaces may in such cases be the result of normal structures with characteristics similar to pericardial fluid, such as fat surrounding the heart. This hypothesis was proposed for this investigation.

Computerised tomography is a reliable noninvasive means of distinguishing pericardial fluid from fat since the fluid produces positive computerised tomography values and the fat produces negative computerised tomography values. ${ }^{4} 5$ Therefore, computerised tomography was used in the present study to assist in determining the cause of the extra echo spaces detected echocardiographically. 


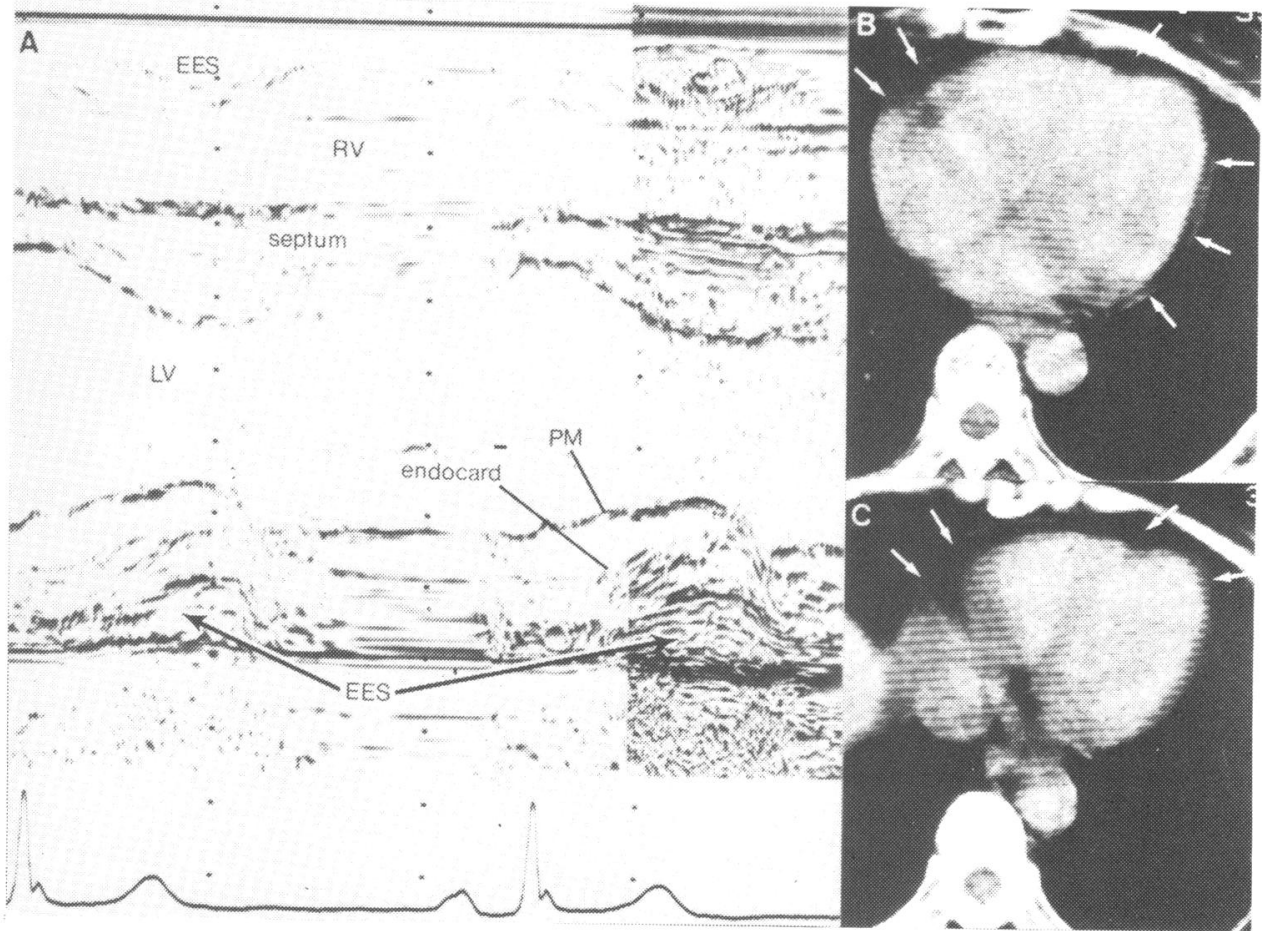

Fig. 1 A 70-year-old fapanese woman with ischaemic heart disease and hypertension. (A) Her echocardiograms consistently showed dense extra echo spaces both anteriorly and posteriorly which became relatively echo free using automatic gain control. (B) and (C) Computerised tomography scans show a linear band encircling the heart, representing the thin pericardium-epicardium (white arrows). There is no evidence of pericardial effusion. Between the thin pericardium-epicardium and the heart, a broad lucent-appearing zone indicates subepicardial fat anteriorly and posteriorly. The extra echo spaces on the echocardiogram are attributed to fat, not to an effusion (courtesy of Dr Satoshi Ohta). EES, extra echo spaces; $R V$, right ventricular cavity; septum, interventricular septum; $L V$, left ventricular cavity; PM, papillary muscle; endocard, endocardium.

\section{Method}

From January 1980 to April 1981, 844 in- and outpatients of the International Goodwill Hospital received 1497 echocardiographic examinations. The echocardiography was performed by one of the authors $(\mathrm{MH})$ using an Aloka model SSD-110 unit, and included strip chart tracings. In each case, a 2.25 $\mathrm{MHz}$ transducer was placed at the fourth intercostal space at the left parasternal region for M-mode scanning from the aorta to the cardiac apex. Technical errors which can produce "false echo-free spaces" were thus avoided.$^{67}$ Automatic gain control was used in all tracings to delineate clearly the border zone echoes. The posteriorly located extra echo spaces imaged here were those behind the posterior wall of the left ventricle, and were identified in the vicinity of scarcely visible mitral echoes.

After omitting 144 cases with poorly identified posterior left ventricle walls, the remaining 700 cases were analysed. Variations in sizes and locations of the extra echo spaces were assessed during interpretation of the echocardiograms. When repeated recordings were made of the same patients, extra echo spaces were consistently observed. The posteriorly located extra echo spaces were categorised as systolic, and systolic and diastolic combined.

Among the 700 cases, the 50 most recently examined were studied using a Varian model V-360-3 computerised tomography scanner, with 3.6 second scan. Without contrast media, computerised tomography scans were made of the heart at 12 different levels, from aorta to the cardiac apex. The computerised tomography values were recorded in the region of the heart below the level of the mitral valve. The $\mathbf{5 0}$ patients ranged in age from 37 to 84 years, with a mean of 64 years. There were 29 women and 21 men. Among the 50 patients, 21 had ischaemic heart disease with or without hypertension: 10 with cardiomyopathy, four with atrial septal defects, three 
with valvular heart disease, two with collagen disease, and one each, pulmonary hypertension, aortitis, pericarditis, renal hypertension, and arrhythmia. The remaining five cases did not have cardiac disease.

Height weight ratios were calculated for each patient according to Broca's index.

\section{Results}

Anteriorly located extra echo spaces were observed in 607 cases (86.7\%). In $229(32.7 \%)$, posteriorly located extra echo spaces occurred only during systole. In 200 cases (28.5\%), extra echo spaces occurred in both systole and diastole (Table). The 429 cases with posterior extra echo spaces all had anterior extra echo spaces.

Table Extra echo spaces by age

\begin{tabular}{|c|c|c|c|c|}
\hline \multirow[t]{3}{*}{ Decade } & \multirow{3}{*}{$\begin{array}{l}\text { No. of } \\
\text { patients }\end{array}$} & \multicolumn{3}{|c|}{ Extra echo spaces } \\
\hline & & \multirow[t]{2}{*}{ Anterior } & \multicolumn{2}{|l|}{ Posterior } \\
\hline & & & Systolic & $\begin{array}{l}\text { Systolic/ } \\
\text { diastolic }\end{array}$ \\
\hline $\begin{array}{c}0-9 \\
10-19 \\
20-29 \\
30-39 \\
40-49 \\
50-59 \\
60-69 \\
70-79 \\
80-89 \\
\text { Total }\end{array}$ & $\begin{array}{r}9 \\
32 \\
57 \\
98 \\
158 \\
139 \\
101 \\
82 \\
24 \\
700\end{array}$ & $\begin{array}{c}0 \\
15(46.8 \%) \\
33(57 \cdot 8 \%) \\
79(80.6 \%) \\
146(92 \cdot 4 \%) \\
135(97 \cdot 1 \%) \\
97(96.0 \%) \\
78(95 \cdot 1 \%) \\
24(100 \%) \\
607(86.7 \%)\end{array}$ & $\begin{array}{l}0 \\
0 \\
7(12 \cdot 2 \%) \\
25(25 \cdot 5 \%) \\
69(43 \cdot 6 \%) \\
60(43 \cdot 1 \%) \\
42(41 \cdot 5 \%) \\
23(28 \cdot 0 \%) \\
3(12 \cdot 5 \%) \\
229(32 \cdot 7 \%)\end{array}$ & $\begin{array}{l}0 \\
0 \\
0 \\
13(13 \cdot 2 \%) \\
40(25 \cdot 3 \%) \\
49(35 \cdot 2 \%) \\
37(36 \cdot 6 \%) \\
42(51 \cdot 2 \%) \\
19(79 \cdot 1 \%) \\
200(28 \cdot 5 \%)\end{array}$ \\
\hline
\end{tabular}

All 50 patients who had computerised tomography scans had both anterior and posterior extra echo spaces echocardiographically. In 21 cases, posteriorly located extra echo spaces occurred only during systole. In the remaining 29 cases, extra echo spaces occurred in both systole and diastole. The dense, broad, band-like echo occurring behind the posterior wall of the left ventricle is that of "pericardium"; its systolic movements were nearly imperceptible in 11 cases, suggesting the presence of pericardial effusions. ${ }^{1}$ In the remaining 38 cases, minimal "pericardial" motion occurred parallel to the systolic movements of the posterior wall of the left ventricle.

Computerised tomography disclosed fat around the hearts of the 50 patients scanned, accounting for the extra echo spaces on their echocardiograms. The computerised tomography values of these fat deposits varied from -36 to -115 , with a mean of -87 , clearly distinct from those of pericardial effusions which had positive computerised tomography values from 0 to +40 , depending upon the components of the effusions. ${ }^{5}$ The fat deposits were subepicardial anteriorly in all 50 cases. They were also posteriorly subepicardial in 18 cases. The relatively few cases with posterior rather than anterior subepicardial fat were the result either of a masking effect by the overlying lung or of vigorous movements of the posterior wall of the left ventricle. It did not necessarily indicate that less fat was present posteriorly. The computerised tomography of the 50 patients indicated that six had both pericardial effusions and fat. Imperceptible "pericardial" movement was encountered on the echocardiograms of five of the six, and in six other cases with only fat surrounding their hearts. This suggests that imperceptible "pericardial" movement is not a reliable criterion for diagnosing pericardial effusions.

Posterior subepicardial fat was clearly visible when pericardial effusions were present, but as the effusions resolved, the posteriorly located fat was rendered indistinct for the reasons previously mentioned. Computerised tomography could clearly distinguish fat from effusions, but echocardiography could not clearly differentiate the extra echo spaces caused by the two conditions.

According to Broca's index, seven patients were overweight and two were underweight, but there were no significant correlations between the extra echo spaces and the patients' obesity.

\section{Discussion}

Echocardiography has facilitated the diagnosis of pericardial effusions. In 1972, an attractive hypothesis was advanced that the extent of pericardial effusions could be estimated according to the sizes and shapes of their "echo-free spaces". 1 Though many investigators have tested this hypothesis, reports of follow-up studies of pericardial effusions are unavailable, particularly any with evidence of complete resolution of "echo-free spaces" after treatment. An illustrative case of acute myocarditis with pericardial effusion was reported in the first ${ }^{1}$ but not the subsequent editions of one text. ${ }^{89}$ Others have reported cases before and after dialysis ${ }^{1011}$ and one of tuberculous pericarditis with effusion. ${ }^{12}$ Though "echofree spaces" of these cases decreased in extent, they did not resolve completely. Constrictive pericarditis was the only explanation offered for residual "echofree spaces". 12

Horowitz et al. ${ }^{13}$ reported anterior "echo-free spaces" despite pericardial fluid in volume of $16 \mathrm{ml}$ or less, confirmed at surgery. They pointed out that anterior "echo-free spaces" caused by pericardial effusions are most unusual. The nature and cause of such "echo-free spaces" have not been explained. One investigator was reluctant to diagnose pericardial effusions in patients with only anterior "echo-free spaces". 189 A small pericardial effusion, however, often forms anterior to the heart rather than posteriorly because of the effect of gravity on the heart 


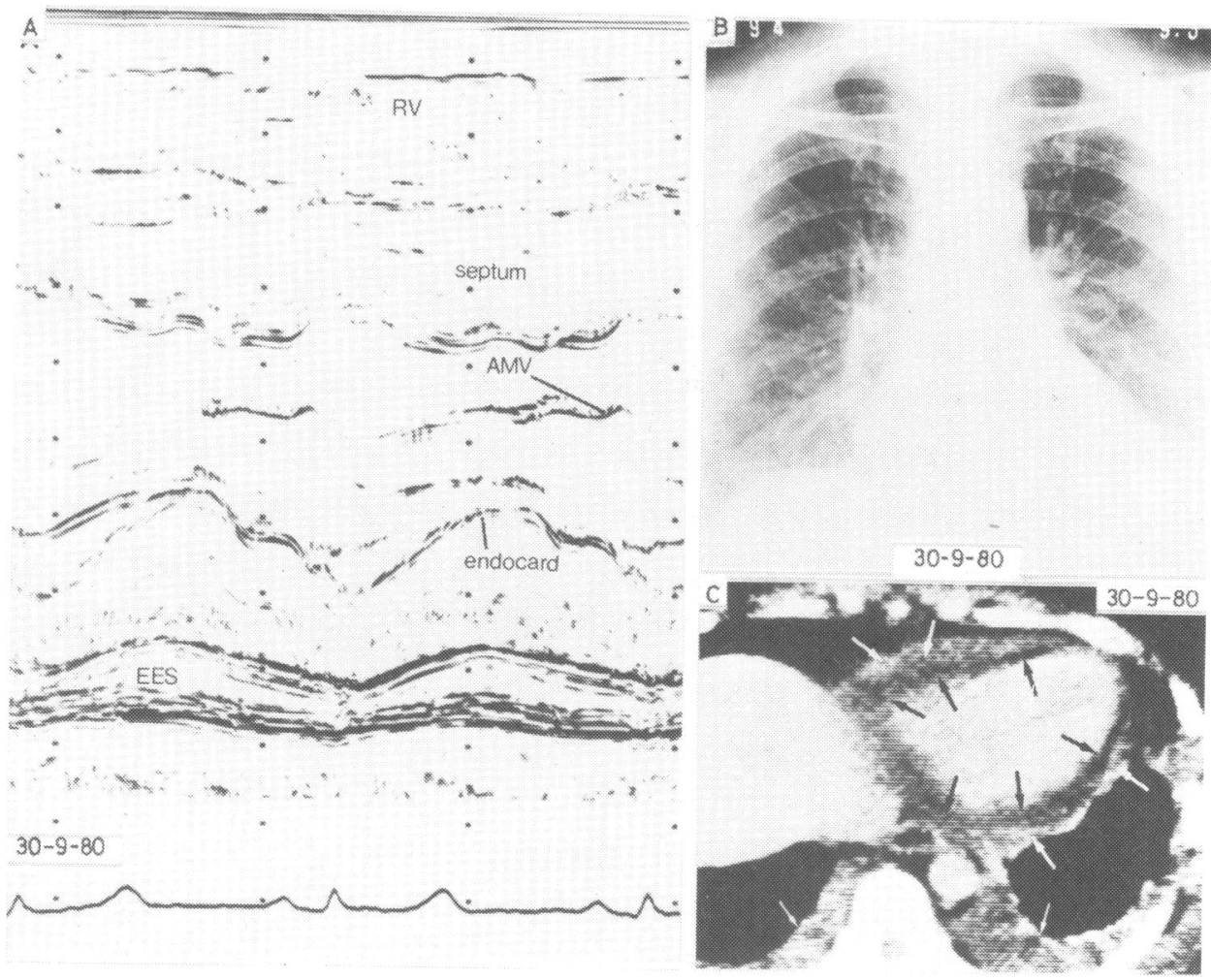

Fig. 2 A 53-year-old fapanese woman with congestive heart failure caused by renal hypertension. (A) Her echocardiogram (speed: $100 \mathrm{~mm} / \mathrm{s}$ with automatic gain control) shows that the anterior mitral valve echoes are scarcely visible. The image in the posterior portion of this echocardiogram represents the posterior wall of the left ventricle. There are distinct extra echo spaces posteriorly, and behind the posterior one is a dense, broad, band-like echo whose minimal movement is parallel to the systolic movements of the left ventricle, suggesting a moderate pericardial effusion. (B) A posteroanterior (PA) chest radiograph the same day as echocardiography shows an increased cardiothoracic ratio and bilateral pleural effusions. (C) A computerised tomography scan the same day as echocardiography and chest radiography shows a shadow surrounding the heart, indicative of moderate pericardial effusion (white arrows), much greater in extent anteriorly than posteriorly because of posterior shift of the heart caused by gravity. The radiolucent zone surrounding the heart is caused by subepicardial fat (black arrows). Since the posteriorly located fat is much greater in extent than the effusion, a greater portion of the posterior extra echo spaces on echocardiography is caused by fat than by effusion. The bilateral pleural effusions detected radiographically were confirmed by computerised tomography (small white arrows). RV, right ventricular cavity; septum, interventricular septum; AMV, anterior mitral valve; endocard, endocardium; EES, extra echo spaces.

(Fig. 2, 3, and 4). Ideally, echocardiography should detect such a small pericardial effusion.

Posterior "echo-free spaces" are attributed to pericardial effusions based on assumptions that a dense, broad, band-like echo behind the posterior wall of the left ventricle is of pericardial origin, and the curvilinear echo above the "echo-free spaces" is caused by the epicardium. If the former assumption were true, such an echo would not occur with complete absence of the left pericardium. In each of four cases of complete absence of the left pericardium, we observed identical dense broad band-like echoes behind posterior wall of the left ventricle ${ }^{14}$ (Fig. 5).
These echoes could not have represented the pericardium. Other investigators have reported similarly. ${ }^{15-17}$ The epicardium consists of a single layer of mesothelial cells only 5 to $6 \mu$ thick, and it would be unusual for such a thin layer to produce a recognisable echo on echocardiography. There is some connective and areolar tissue beneath the epicardium, ${ }^{2}$ and the epicardium and this connective tissue apparently play a role in producing the so-called "epicardial echo". The areolar tissue contains varying amounts of fat, blood vessels, and nerves. Anatomically, the thickness of this tissue has not been measured, but radiographically, it is $2 \mathrm{~mm}$ thick in $75 \%$ of adults. ${ }^{18}$ The 


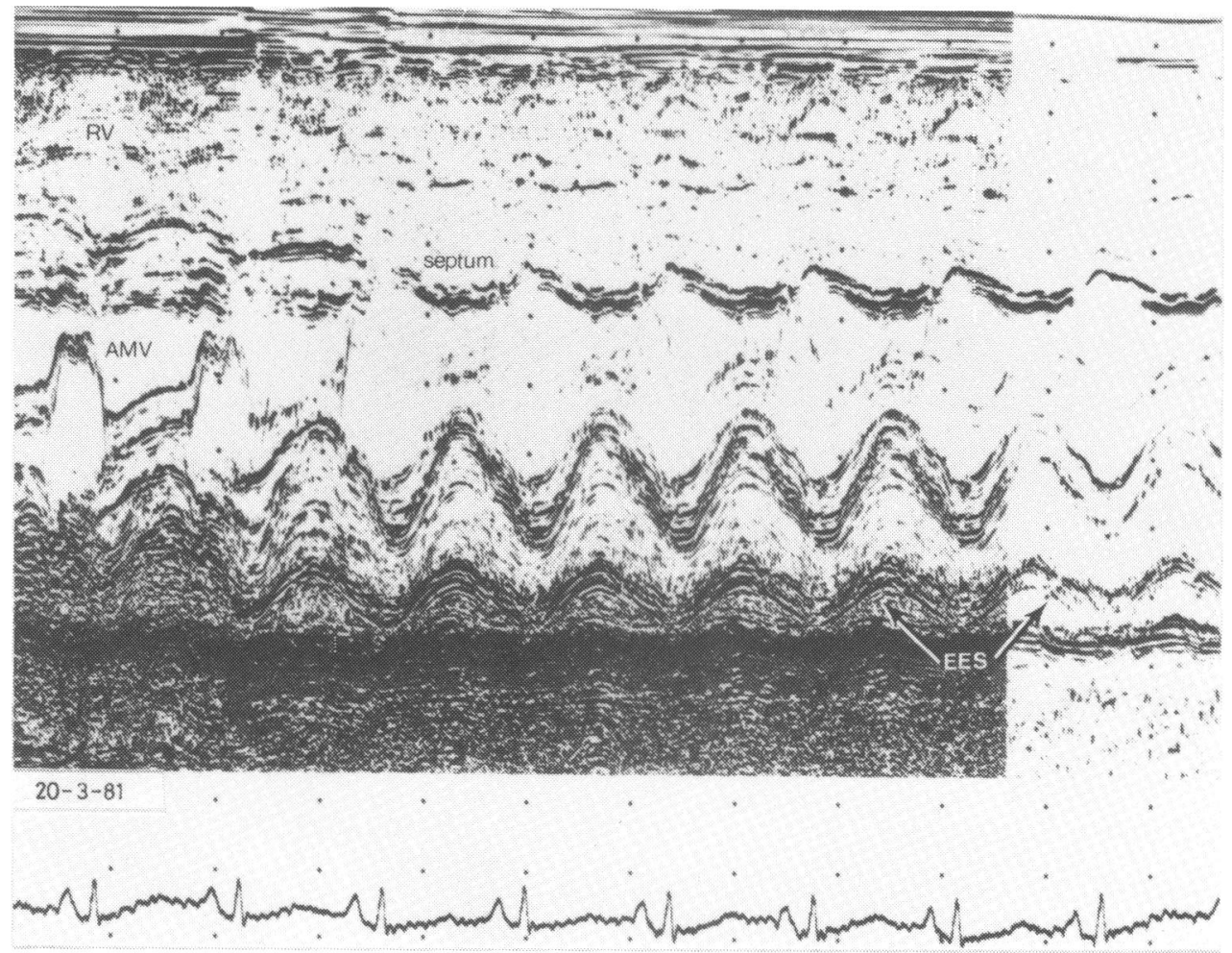

Fig. $3 M$-mode scan of the same patient six months later. Continuity of the ventricular septum and the anterior mitral valve echoes is normal. Posteriorly located extra echo spaces are echo dense and become echo free with automatic gain control. The dense broad band-like echo behind the posterior wall of the left ventricle moves minimally, parallel with systolic motion of the left ventricle. The anterior and posterior extra echo spaces are indistinguishable from those seen six months earlier. $R V$, right ventricular cavity; septum, interventricular septum; $A M V$, anterior mitral valve; $E E S$, extra echo spaces.

subepicardial fat can be appreciable in quantity and can mimic cardiomegaly on chest radiography. Chest surgery, however, is necessary to prove the nature of such apparent cardiomegaly. ${ }^{19} 20$ Accordingly, it may be assumed that a thick layer of subepicardial fat can produce extra echo spaces on echocardiography. This is supported by results of experimental echocardiography in which two or more linear echoes issued from a strip of myocardium containing subepicardial fat. ${ }^{21}$ The first linear echo was said to be of epicardial origin, but the investigators did not speculate about the origin of the later echoes. We believe that the first linear echo originated from the border between the epicardium and areolar tissue. It is followed by an "echo-free space" caused by fat. The second linear echo in the "echo-free space" is attributed to the coronary vessels. The last linear echo is probably the result of the border between areolar tissue and myocardium.

In the present study, differences in computerised tomography values clearly showed that the extra echo spaces were the result of pericardial and/or subepicardial fat, rather than of effusions. Accordingly, the 8 dense broad band-like echo behind the posterior wall

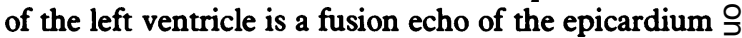
and subepicardial tissue, the pericardium, and the pleura. The former two tissues comprise the potential pericardial cavity which is not visualised except in $N$ cases of effusions. Anterior to the dense broad bandlike echo is another extra echo space caused by subepicardial fat, and there may be scattered miliary $\omega$ echoes resulting from coronary vessels and nerves. The superior portion of the extra echo spaces is 6 demarcated by a curvilinear echo produced at the $\mathbb{\Phi}$ junction between subepicardial fat and myocardium. ? This curvilinear echo has been misinterpreted as an $T$ epicardial echo. The fat-producing extra echo spaces were thought to represent the pericardial cavity $\stackrel{\square}{\Phi}$ rendered visible by effusions. The concept that fat $\stackrel{\AA}{\perp}$ produces extra echo spaces had not been reported $\bar{Q}$ 


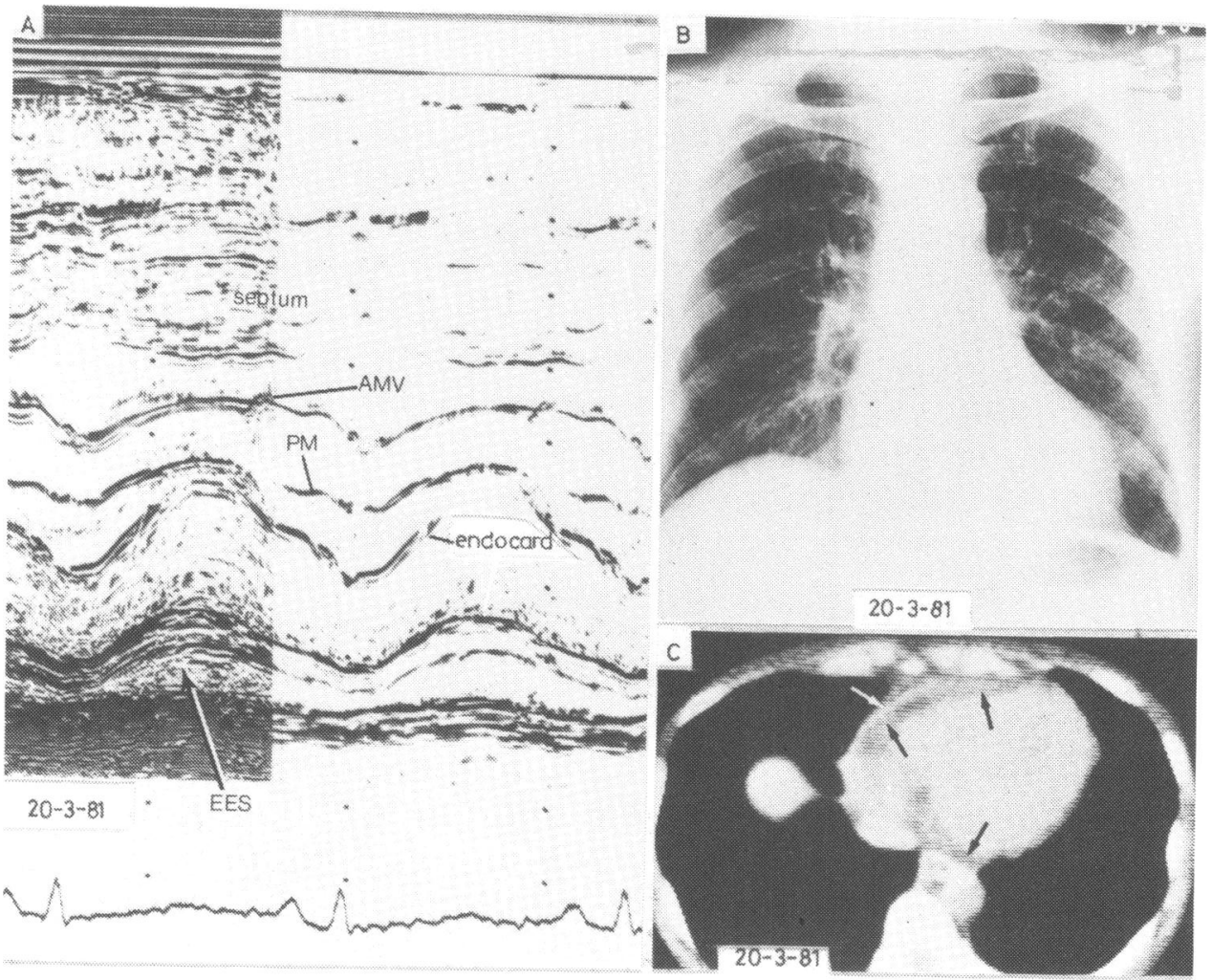

Fig. 4 (A) High speed echocardiogram the same day as Fig. 3 shows an extra echo space behind the posterior wall of the left ventricle which became "echo-free" with the aid of automatic gain control. Size and contour were unchanged since the initial examination of 30-9-80. The extra curvilinear echo in the "echo-free spaces" is probably the result of coronary vessels. (B) PA chest radiograph of 20-3-81 shows a decrease in cardiac size and no pleural effusion. (C) A computerised tomography scan on 20-3-81 shows that the previously observed pericardial effusion resolved except for a minimal effusion near the anterior portion of the heart (white arrow), causing an increased width of pericardium-epicardium silhouette. The posteriorly located subepicardial fat is no longer visible, because of resolution of the pericardial effusion. Anterior subepicardial fat (black arrows) remains visible and is greater in extent than the pericardial effusion. Though the posterior subepicardial fat is not visible on the computerised tomography scan, there is no evidence of pericardial effusion posteriorly. Thus, the posterior extra echo space visualised echocardiographically is not the result of a pericardial effusion. This is a good example of residual extra echo spaces after the treatment and resolution of a pericardial effusion. Septum, interventricular septum; $A M V$, anterior mitral valve; PM, papillary muscle; endocard, endocardium; EES, extra echo spaces.

except for brief suggestions in texts, ${ }^{1} 8922$ relating to anterior extra echo spaces. Assuming that fat surrounding the heart can cause extra echo spaces, the residual "echo-free spaces" still visible after successful treatment of pericardial effusions are easily explained.

Increased accumulation of subepicardial fat occurs during the aging process, ${ }^{3}$ as was observed in the present study. Patients under 9 years of age had neither anterior nor posterior extra echo spaces. Adolescents had only anterior extra echo spaces. Posterior extra echo spaces were observed in only $12 \cdot 2 \%$ of patients between the ages of 20 and 29 years. Both anterior and posterior extra echo spaces increased in frequency after the age of 29 years. Complete resolution of the extra echo spaces of a patient with acute myocarditis has been reported ${ }^{1}$ and was probably a young individual, without residual extra echo spaces caused by excessive fat around the heart. In our series of pericardial effusions, only one patient, a 43-year-old emaciated Japanese man with tuberculous pericardial effusion, displayed complete resolution of his extra echo spaces. Corticosteroids can enhance the accumulation of subepicardial fat, ${ }^{3}$ but none of the patients in the present study received such treatment.

In a variety of heart diseases, posteriorly located 
(A)

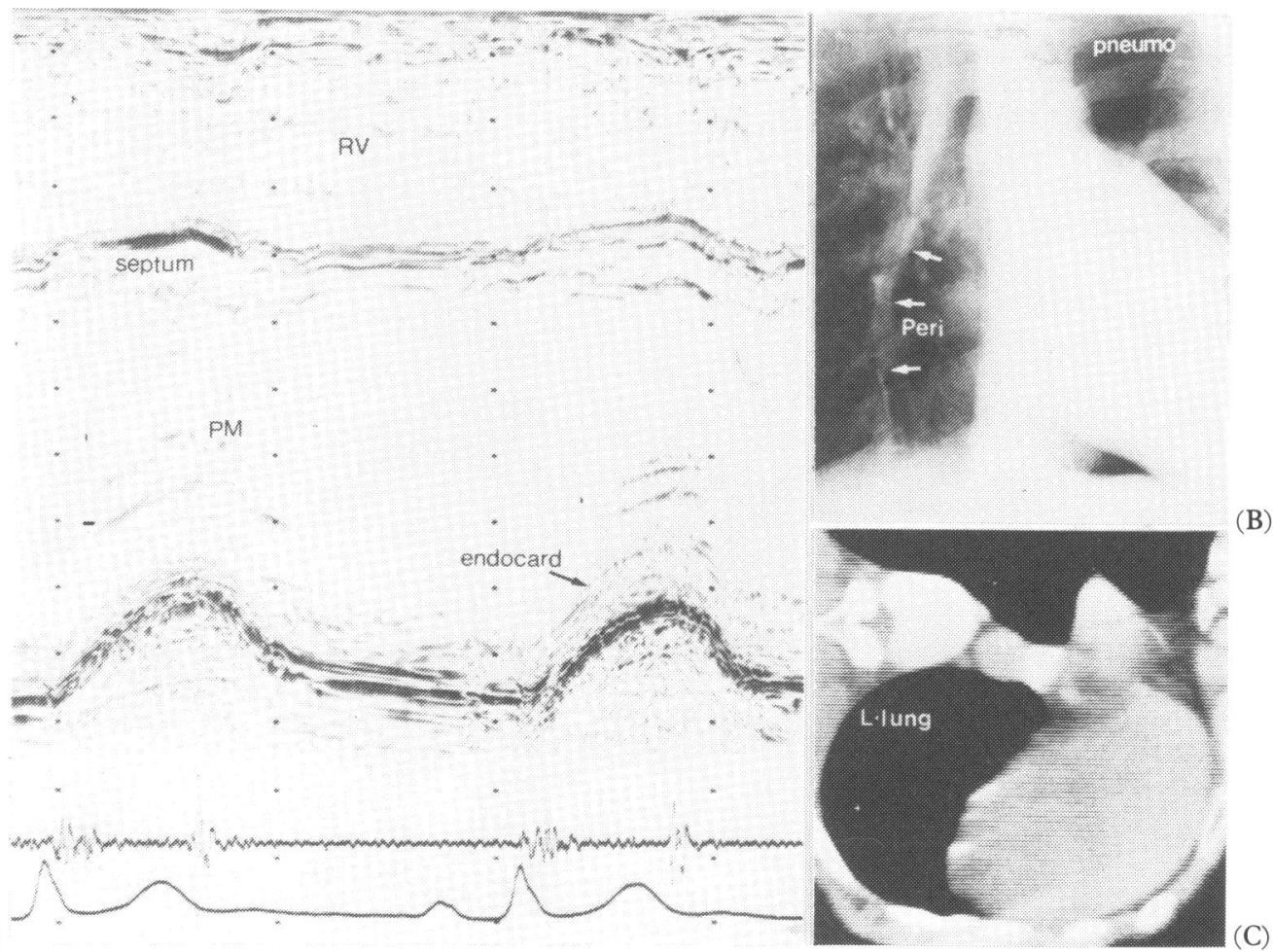

Fig. 5 A 33-year-old fapanese man with congenital complete absence of the left pericardium. (A)

Echocardiogram showing hyperkinetic movements of posterior wall of the left ventricle and paradoxical movement of the ventricular septum - the usual manifestations of this abnormality. The dense broad band-like echo behind the posterior wall of the left ventricle is indistinguishable from that normally seen. Since the left pericardium is absent, the dense broad band-like echo cannot be a pericardial echo. It is attributed to thickening of the epicardium and the connective tissue beneath it. (B) PA chest radiograph after a left pneumothorax resulting in a pneumopericardium on the right (white atrows) caused by absence of the lefi pericardium-parietal pleura. (C) A computerised tomography scan in the left lateral decubitus position shows that the heart descends to the left thoracic wall, indicating complete absence of the left pericardium. There were no densities indicative of pericardium-epicardium on the left side at any level of the computerised tomography scans. RV, right ventricular cavity; septum, interventricular septum; PM, papillary muscle; endocard, endocardium; Peri, pericardium; pneumo, pneumothorax; L lung, left lung.

extra echo spaces are reflected by echo-dense or echofree patterns. No explanations have been offered for the cause of such spaces, ${ }^{23}$ though in echocardiographic texts, similar findings have been reported to be the result of factors other than pericardial effusions. In the majority of such cases, improper gain damping was used, resulting in a poorly demarcated border zone, and the extra echo spaces were overlooked. To image the posterior "echo-free spaces" clearly, the examiner must always anticipate such lesions, using proper gain damping in each case. Automatic gain control enables the "echo-free spaces" to be imaged without much effort. In fact, since our preliminary reports, 2425 the rate at which we have detected extra echo spaces has increased nearly tenfold with the routine use of automatic gain control. The frequencies of posterior extra echo spaces reported in Japanese texts varied from $8.7 \%$ to $49 \%$, with a mean of $\frac{D}{0}$ $24.2 \%^{12} 16^{1726-28}$; whereas, in English texts, their frequencies varied from $5 \%$ to $17 \cdot 2 \%$, with a mean of $\mathrm{N}$ 9\%. ${ }^{9-1129-33}$ The reason for these differences is not $N$ clear, but they probably arise from the use of a variety of echocardiographic units of different manufacture, and the various interests of the investigators involved. A very improbable explanation is that Japanese have more fat around their hearts than Americans. Least likely is the possibility that some of our tracings were made too medially to image the posterior wall of the left ventricle, and that the extra echo spaces in the present study were artefacts. When tracings are made too medially, however, the continuity of the mitral valve echoes is lost, and multiple-layer echoes result. 


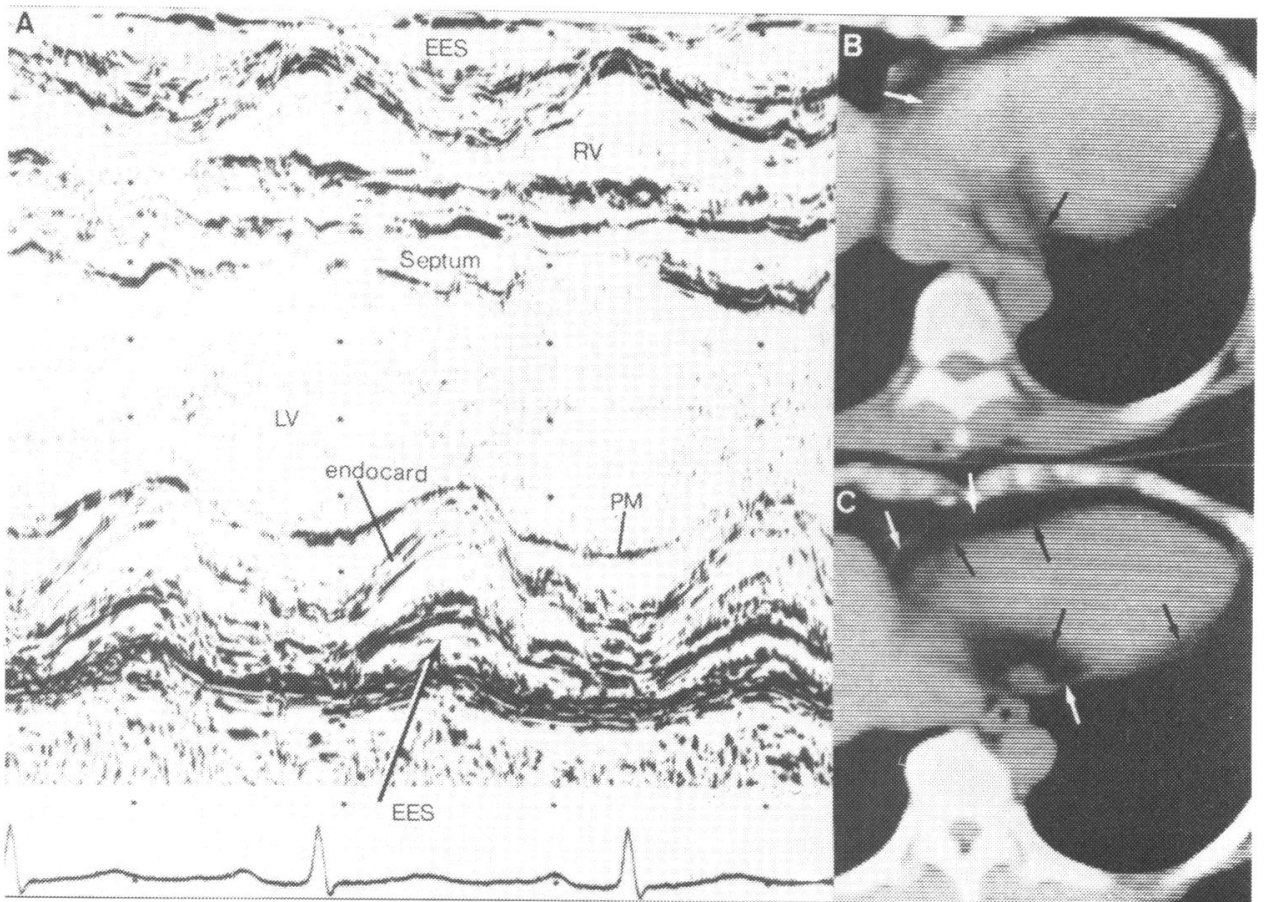

Fig. 6 A 58-year-old fapanese woman with ischaemic heart disease with hypertension. (A) Her echocardiograms consistently showed both anterior and posterior extra echo spaces. Though she was asymptomatic, her chest radiographs indicated that her heart was enlarged. (B) and (C) computerised tomography showed considerable pericardial fat anteriorly, and subepicardial fat both anteriorly and posteriorly (black arrows). The pericardium-epicardium image is thin (white arrows), indicating that there was no pericardial effusion. EES, extra echo spaces; $R V$, right ventricular cavity; septum, interventricular septum; $L V$, left ventricular cavity; $P M$, papillary muscle; endocard, endocardium.

In the present study, we took great care in using M-mode scanning from the aorta to the cardiac apex in each case. Therefore, the extra echo spaces observed could not have been caused by artefacts; they are attributed to fat around the heart.

The present study correlated the computerised tomography images of pericardial and/or subepicardial fat with the extra echo spaces imaged by echocardiography. There is adequate explanation for the extra echo spaces frequently observed in the asymptomatic patient (Fig. 6), and the persistent extra echo spaces observed during follow-up studies of successfully treated pericardial effusions. Thus, fat around the heart can produce extra echo spaces indistinguishable from those of pericardial effusions. The echocardiographic diagnosis of pericardial effusions is therefore difficult, unless they occur in relatively young patients without appreciable fat posteriorly, or in massive effusions which cause pendulum-like cardiac motion.

Finally, the extra echo spaces caused by fat can outline the space between the epicardium and myocardium, but not the pericardial space which is outlined by pericardial effusions. The extra echo spaces can be either echo dense or echo free; thus the term "echo-free spaces" is confusing. For the benefit of students and those beginning as echocardiographers this term should not be used.

The authors thank Dr Walter J Russell for reviewing the manuscript and for his editorial suggestions.

\section{References}

1 Feigenbaum H. Echocardiography. Philadelphia: Lea \& Febiger, 1972: 163-83.

2 Wheater PR, Burkitt HG, Daniels VG. Functional histology. Edinburgh, London, New'York: Churchill Livingstone, 1979: 76-7.

3 Spodick DH. Pericardial disease. Philadelphia: FA Davis, 1976: 14-7.

4 Alfidi RJ, MacIntyre WJ, Meaney TF, et al. Experimental studies to determine application of CT scanning to the human body. Am 7 Roentgenol Radium Ther Nucl Med 1975; 124: 199-207. 
5 Tomoda H, Hoshiai M, Furuya H. Evaluation of pericardial effusion with computed tomography. Am Heart $\mathcal{f}$ 1980; 99: 701-6.

6 Come PC, Riley MF, Fortuin NJ. Echocardiographic mimicry of pericardial effusion. Am $\mathcal{F}$ Cardiol 1981; 47: 365-70.

7 Reeves WC, Ciotola T, Babb JD, Buonocore E, Leaman D. Prolapsed left atrium behind the left ventricular posterior wall: two dimensional echocardiographic and angiographic features. Am f Cardiol 1981; 47: 708-12.

8 Feigenbaum H. Echocardiography. 2nd ed. Philadelphia: Lea \& Febiger, 1976: 419-46.

9 Feigenbaum H. Echocardiography. 3rd ed. Philadelphia: Lea \& Febiger, 1981: 478-504.

10 Felner JM, Schlant RC. Echocardiography. New York: Grune \& Stratton, 1976: 342.

11 Joyner CR. Ultrasound in the diagnosis of cardiovascularpulmonary disease. Chicago: Year Book Publisher, 1974: 113.

12 Machii K, Sakamoto T, Matsuo H, Kato H. Atlas of echocardiography. Tokyo: Chugai-Igaku, 1980: 164-7.

13 Horowitz MS, Schultz CS, Stinson EB, Harrison DC, Popp RL. Sensitivity and specificity of echocardiographic diagnosis of pericardial effusion. Circulation 1974; 50: $239-47$.

14 Yanagisawa N, Honda M. Tazumi K, Higuchi Y, Wada $\mathrm{T}$. Non-invasive diagnosis of pleuro-pericardial defect. Fournal of Cardiography 1979; 9: 189-99.

15 Payvandi MN, Kerber RE. Echocardiography in congenital and acquired absence of the pericardium. Circulation 1976; 53: 86-92.

16 Fujii J. Echocardiography. Tokyo: Nankodo, 1980: 176.

17 Yoshikawa J. Clinical echocardiography. Tokyo: Kanehara, 1979: 378.

18 Stewart JR, Kincaid DW. A new simple radiographic screening method for the diagnosis of pericardial effusion. Proceedings of the Mayo Clinic 1963; 38: 339-44.

19 Tucker DH, Gaylor DH, Jacoby WJ Jr, Sumner RG. Prominence of the left epipericardial fat pad. Am $\mathcal{Y}$ Med 1965; 38: 268-73.
20 James AE Jr, Torrance DJ. Pericardial fat simulating cardiomegaly. Am Heart $\mathcal{f}$ 1965; 69: 93-5.

21 Yoshikawa J, Yanagihara K, Owaki T, et al. Problems in echocardiographic identification of the endocardium, $\overrightarrow{\vec{F}}$ epicardium, and pericardium of the posterior left ventricle. Fapanese F Med Ultrasonic 1977; 38: 268-73.

22 Cohen MV. Correlative atlas of adult cardiac disorders. New York: Futura, 1980: 349.

23 Feigenbaum H. Newer aspects of echocardiography. Circulation 1973; 47: 833-42.

24 Honda M, Watanabe H, Mori S, et al. Re-evaluation of the echo-free space on the echocardiogram. Foumal of Cardiography 1980; 10: 791-800.

25 Wada T, Honda M. Pericardial diseases - echo-free spaces. Medicina Tokyo 1981; 18: 1392-8.

26 Machii K. Clinical echocardiography. Tokyo: ChugaiIgaku, 1973.

27 Kawai N. Practical echocardiography. Tokyo: Nanzando, 1977.

28 Sawayama T, Kawai N, Maeda K. Atlas of cardiography. Tokyo: Chugai-Igaku, 1979.

29 Haft JI, Horowitz MS. Clinical echocardiography. New York: Futura, 1978.

30 Williams RG, Tucker CR. Echocardiographic diagnosis of $\stackrel{2}{=}$ congenital heart disease. Boston: Little, Brown, 1977.

31 Goldberg SJ, Allen ED, Sahn DJ. Pediatric and adoles- $\infty$ cent echocardiography. Chicago: Year Book Medical Pub- ? lishers, 1975.

32 Phillips BJ, Friedewald VE Jr. Manual of echocardiographic techniques. Philadelphia: WB Saunders, 1980.

33 Kield JJ, Schiller NB, eds. Echocardiography case studies: 58 case histories related to echocardiography. New York: Medical Examination Publishing, 1974.

Requests for reprints to Dr Takashi Wada, International Goodwill Hospital 55, 3-chome, Aioi-cho, Naka-ku, Yokohama 231, Japan. 